\title{
Discovering Planetary Nebula Geometries: Explorations with a Hierarchy of Models
}

\author{
Karen A. Huyser ${ }^{* \dagger}$, Kevin H. Knuth ${ }^{\dagger}$, Bernd Fischer ${ }^{\dagger * *}$, Johann \\ Schumann $^{\dagger * *}$, Domhnull Granquist-Fraser ${ }^{\dagger \star}$ and Arsen R. Hajian ${ }^{\S}$ \\ *Stanford Univ. EE Dept, Stanford, CA 94305 USA \& Education Assoc's Program, NASA Ames \\ ${ }^{\dagger}$ NASA Ames Research Center, Moffett Field CA 94035 USA \\ ${ }^{* *}$ Research Institute for Advanced Computer Science \\ ${ }^{\ddagger}$ QSS Group \\ ${ }^{\S}$ US Naval Observatory, Washington DC 20016 USA
}

\begin{abstract}
.
Astronomical objects known as planetary nebulae (PNe) consist of a shell of gas expelled by an aging star. In cases where the gas shell can be assumed to be ellipsoidal, the PN can be easily modeled in three spatial dimensions. We utilize a model that joins the physics of PNe to this geometry and generates simulated nebular images. Hubble Space Telescope images of actual PNe provide data with which the model images may be compared. We employ Bayesian model estimation and search the parameter space for values that generate a match between observed and model images. The forward model is characterized by thirteen parameters; consequently model estimation requires the search of a 13-dimensional parameter space. The 'curse of dimensionality,' compounded by a computationally intense forward problem, makes forward searches extremely time-consuming and frequently causes them to become trapped in a local solution. We find that both the speed and quality of the search can be improved by reducing the dimensionality of the search space.

Our basic approach utilizes a hierarchy of models of increasing complexity. Earlier studies establish that a hierarchical sequence converges more quickly, and to a better solution, than a search relying only on the most complex model. Here we report results for a hierarchy of five models. The first three models treat the nebula as a 2D image, estimating its position, angular size, orientation and rim thickness. The last two models explore its characteristics as a 3D object and enable us to characterize the physics of the nebula. This five-model hierarchy is applied to real ellipsoidal PNe to estimate their geometric properties and gas density profiles.
\end{abstract}

\section{BACKGROUND}

Stars with $0.8-8$ solar masses end their lives as swollen red giants surrounded by cool extended atmospheres. Nuclear reactions in the red giant core create carbon, nitrogen, and oxygen, which are transported by convection to the outer envelope of the atmosphere. As the red giant finally collapses to become a white dwarf, this envelope is expelled from the star to form a planetary nebula $(\mathrm{PN})$ rich in organic molecules. (See Figure 1.) The physics, dynamics, and chemistry of these nebulae are poorly understood and have implications not only for our understanding of the stellar life cycle but also for organic astrochemistry and the creation of prebiotic molecules in interstellar space.

Three-dimensional (3D) PN models are inferred from data consisting of images obtained with the Hubble Space Telescope (HST) [1]. We employ Bayesian model estima- 


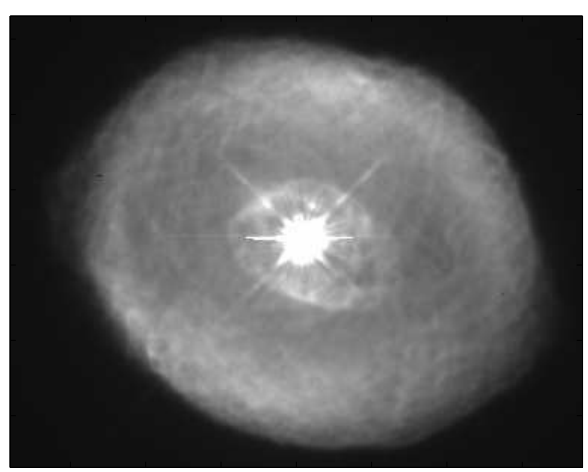

FIGURE 1. Planetary Nebula IC418, the Spirograph Nebula.

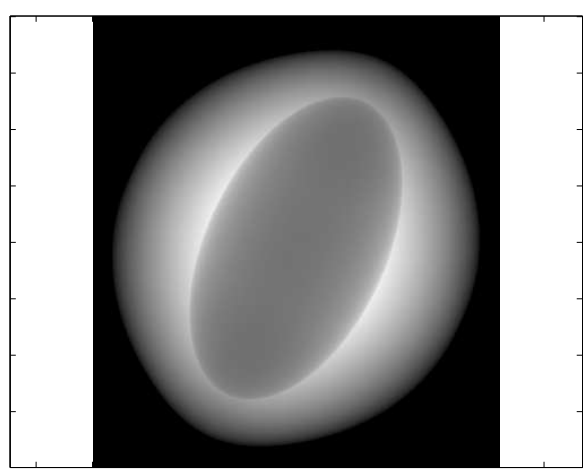

FIGURE 2. Synthetic Nebula ibpes1.

tion using a parameterized physical model of the nebula, which incorporates much prior information about the known physics of how the PN is illuminated by ionizing radiation from the central star. The model captures the nebula's shape, orientation, inclination to the line of sight, and 3D mass distribution. The 2D projection is sensitive to variations in the $3 \mathrm{D}$ parameters, enabling us to learn the nebular structure from the data.

\section{THE HIERARCHY}

Knuth and Hajian [2] developed a hierarchy of models that allow the quick capture of a critical subset of the model parameters. Gauss, SigHat, and SigHat2 are two-dimensional models, while FastSES and IBPES are three-dimensional. Gauss captures the center position and general extent, SigHat captures the eccentricity and orientation, and SigHat2 captures the shell thickness. These two-dimensional models significantly decrease the analysis time and increase the accuracy of the final results, in part by assuring that the final solutions are reasonable. FastSES assumes the PN is made of two concentric, co-axial, equi-eccentric prolate ellipsoids. It refines the SigHat2 estimate of the shell thickness and captures an initial estimate of $\xi$, the luminosity-density parameter. IBPES is the full physical model and captures four parameters used to describe the 3D density profile of the PN and the inclination of the nebula to the line of sight. Figure 2 is a synthetic PN generated with IBPES.

\section{The 2D Models-A Review}

The Gauss model describes the PN image as a 2D circular Gaussian distribution

$$
G(x, y)=I_{o} \exp \left[-\frac{\left(x-x_{o}\right)^{2}+\left(y-y_{o}\right)^{2}}{2 \sigma^{2}}\right] .
$$

Gauss estimates four values: $I_{o}$, which is the overall intensity of the image, $\left(x_{o}, y_{o}\right)$, which are the coordinates of the center of the nebula, and $\sigma$, which is the nebula's overall 
extent. These quantities are basic to any image of any single object and necessarily belong to the simplest model. Figure 3, top left, is an example of a Gauss solution.

Two significant elaborations are made in order to create the SigHat model. First, the circular Gaussian is made elliptical by replacing $\sigma$ by $\sigma_{x}$ and $\sigma_{y}$. Second, the Gaussian is replaced by a sigmoid-hat function, which rises from zero to one, plateaus, and falls symmetrically back to zero. The SigHat function is

$$
S(x, y)=I_{o}\left(1-\frac{1}{1+\exp \{-\lambda[r(x, y)-1]\}}\right)
$$

where

$$
\begin{gathered}
r(x, y)=\left(C_{x x}\left(x-x_{o}\right)^{2}+2 C_{x y}\left(x-x_{o}\right)\left(y-y_{o}\right)+C_{y y}\left(y-y_{o}\right)^{2}\right), \text { and } \\
C_{x x}=\frac{\cos ^{2} \omega}{\sigma_{x}^{2}}+\frac{\sin ^{2} \omega}{\sigma_{y}^{2}}, \quad C_{x y}=\left(\sigma_{y}^{-2}-\sigma_{x}^{-2}\right) \sin \omega \cos \omega, \quad C_{y y}=\frac{\sin ^{2} \omega}{\sigma_{x}^{2}}+\frac{\cos ^{2} \omega}{\sigma_{y}^{2}}
\end{gathered}
$$

Variables $I_{o}, x_{o}$ and $y_{o}$ are as before, $\omega$ is the orientation of the PN in the plane of the sky, $\sigma_{x}$ and $\sigma_{y}$ are the major and minor semi-axes of the ellipse, and $\lambda-$ a nuisance parameter - is the e-folding length of the intensity falloff at the edge of the PN.

SigHat2 models the image as a difference of two sigmoidal hat functions

$$
T(x, y)=I_{+} S_{+}(x, y)-I_{-} S_{-}(x, y) .
$$

$S_{+}(x, y)$ and $S_{-}(x, y)$ are the SigHat functions in (2). The elliptical 'hats' defined by $S_{+}$ and $S_{-}$are concentric, coaxial, and equi-eccentric but have unique semi-axes and falloff rates, $\sigma_{x+}, \sigma_{y+}, \lambda_{+}$and $\sigma_{x_{-}}, \sigma_{y_{-}}, \lambda_{-}$. The equi-eccentric constraint is enforced through use of a thickness ratio, $\Delta$, applied with

$$
\sigma_{x-}=\Delta \cdot \sigma_{x+} \quad \text { and } \quad \sigma_{y-}=\Delta \cdot \sigma_{y+} .
$$

Recall eccentricity is $e^{2}=\left(\sigma_{x-}^{2}-\sigma_{y-}^{2}\right) / \sigma_{y-}^{2}=\left(\sigma_{x+}^{2}-\sigma_{y+}^{2}\right) / \sigma_{y+}^{2}$. See Knuth and Hajian [2] for a complete development of the 2D models.

\section{The 3D Models}

In FastSES and IBPES, the PN is modeled as a prolate spheroidal shell of gas. Since the shell is optically thin at visible wavelengths, the visible intensity of light from the nebula is proportional to the integral along the line of sight of the gas density squared. Consequently, the densest parts of the nebula are visually the brightest.

FastSES, which stands for Fast Scaled Ellipsoidal Shell, describes the PN as two concentric, coaxial, equi-eccentric prolate spheroids with a uniform density gas between them. Let the center of the ellipsoids be $\left(0, y_{o}, z_{o}\right)$, the semi-axes be $a$ and $b$, the orientation $\omega$, and the inclination $\boldsymbol{l}$. For the plane of the sky in the $y z$-plane and for $x$ the line of sight, the distance $d_{x}$ through an oriented and inclined prolate spheroid is given by

$$
d_{x}=\sqrt{B^{2}-4 A C} / A
$$


where

$$
\begin{aligned}
A= & b^{2} \cos ^{2} \imath+a^{2} \sin ^{2} \imath, \quad \hat{A}=b^{2} \sin ^{2} \imath+a^{2} \cos ^{2} \imath, \\
B= & 2 \cos \imath\left(b^{2}-a^{2}\right)\left(\sin \imath \sin \omega\left(y-y_{o}\right)+\sin \imath \cos \omega\left(z-z_{o}\right)\right), \\
C= & \left(\hat{A} \sin ^{2} \omega+a^{2} \cos ^{2} \omega\right)\left(y-y_{o}\right)^{2}+\left(\hat{A} \cos ^{2} \omega+a^{2} \sin ^{2} \omega\right)\left(z-z_{o}\right)^{2} \\
& +2\left(b^{2}-a^{2}\right)\left[\sin \imath \sin \omega\left(y-y_{o}\right)\right]\left[\sin \imath \cos \omega\left(z-z_{o}\right)\right]-a^{2} b^{2} .
\end{aligned}
$$

An imaginary solution to (7) indicates a $(y, z)$ position outside the ellipsoid and, therefore, a zero value for $d_{x}$. The image brightness of pixel $(y, z)$ is given by

$$
F(y, z)=I_{o} n^{2}\left[d_{x o}-d_{x i}\right]
$$

where $n$ is the number density of radiating particles in the gas between the ellipsoids and where $d_{x o}$ and $d_{x i}$ are the line-of-sight distances through the outer and inner ellipsoids, respectively.

IBPES, which stands for Ionization Bounded Prolate Ellipsoidal Shell, is a physicsbased model introduced by Aaquist and Kwok [3] based upon work by Masson [4, 5]. IBPES adds two specific assumptions to those of FastSES. First, it assumes the PN is ionization-bounded, which means that all the ionizing radiation from the central star is absorbed before it reaches the outer boundary of the shell. Thus the ionization boundary comprises the outer boundary of the visible portion of the nebula. Second, it replaces the assumption of an outer ellipsoid with an explicit formula for the gas density. A typical nebula has been compressed radially by hot winds from the central star and may exhibit latitudinal density variations from any of a variety of causes. IBPES models the density $n$ as a separable product of functions of the spherical polar variables

$$
\begin{aligned}
n(r, \theta, \phi) & =n_{o} \eta_{\phi}(\phi) \eta_{r}(r) \eta_{\theta}(\theta) \\
& =n_{o} \eta_{\phi}\left(\frac{r}{R_{i}(\theta)}\right)^{\gamma} \cdot \begin{cases}{\left[\beta+(1-\beta)\left(\frac{2 \theta}{\pi}\right)^{\alpha}\right],} & 0 \leq \theta \leq \frac{\pi}{2} \\
{\left[\beta+(1-\beta)\left(\frac{2 \pi-2 \theta}{\pi}\right)^{\alpha}\right],} & \frac{\pi}{2} \leq \theta \leq \pi\end{cases}
\end{aligned}
$$

where $n_{o}$ and $\eta_{\phi}$ are constants and $R_{i}(\theta)$ is the radial distance from the star to the inner prolate spheroid at latitude $\theta$. Thus radial density variations are modeled as a power law with exponent $\gamma$, while latitudinal density variations - which dramatically affect the shape of the outer ionization boundary-are modeled by a pole-to-equator ratio $\beta$ and a latitudinal density gradient $\alpha$. Density is independent of longitude $\phi$. For semi-major axis $a$, semi-minor axis $b$, and eccentricity $e^{2}=\left(a^{2}-b^{2}\right) / a^{2}$, the radius at latitude $\theta$ is $R_{i}^{2}(\theta)=b^{2} /\left(1-e^{2} \cos ^{2} \theta\right)$.

Ionization-boundedness is equivalent to the assumption that all ionizing photons from the central star are absorbed and re-radiated by the nebula. The energy absorbed can be written as $d L=\alpha_{B} n^{2}(r, \theta, \phi)\left[r^{2} d r d \Omega\right]$ and integrated over $r$ to find the energy radiated per unit solid angle

$$
\frac{d L}{d \Omega}=\alpha_{B}\left[n_{o} \eta_{\phi} \eta_{\theta}(\theta)\right]^{2} \int_{R_{i}}^{R_{o}}\left(\frac{r}{R_{i}}\right)^{2 \gamma} r^{2} d r
$$




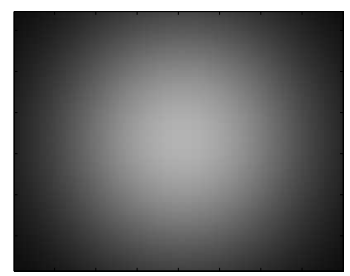

Gauss

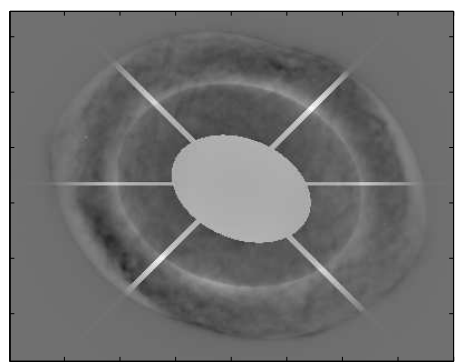

Difference

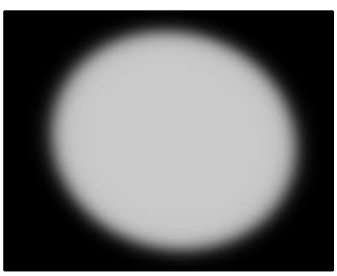

SigHat

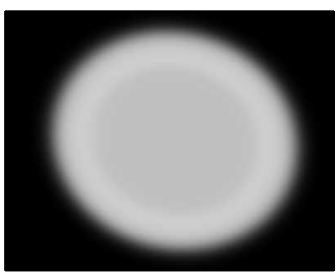

SigHat2

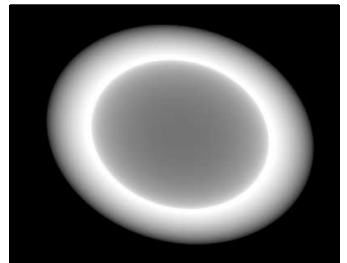

FastSES

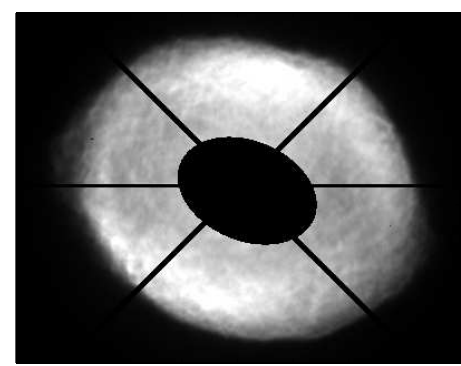

IC418, masked

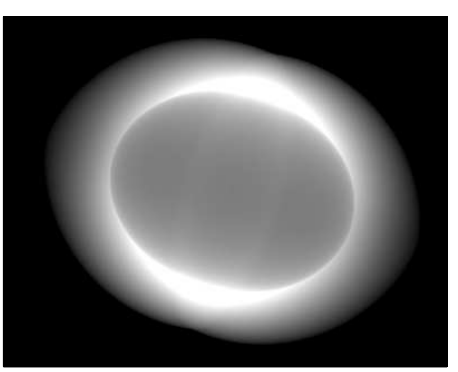

IBPES

FIGURE 3. A Sequence of Models for IC418. On top, left to right, are shown the solutions from Gauss, SigHat, SigHat2, and FastSES. Bottom right is the solution from IBPES. Bottom center is the HST image (the data) after masking out the central star and diffraction spikes. Bottom left is the difference image-IBPES minus the data-in which the medium grey background is zero while black or white areas indicate a large negative or positive difference, respectively.

where $\alpha_{B}$ is an absorption coefficient. $d L / d \Omega$ is constant because the central star provides spherically symmetric illumination to the space around it. For $\gamma \neq 1.5$, integration over $r$ gives

$$
\begin{aligned}
R_{o}(\theta) & =R_{i}(\theta)\left\{1+\left(\frac{1}{\alpha_{B} n_{o}^{2} \eta_{\phi}^{2}} \frac{d L}{d \Omega}\right)\left(\frac{-2 \gamma+3}{R_{i}^{3}(\theta) \eta_{\theta}^{2}(\theta)}\right)\right\}^{\frac{1}{-2 \gamma+3}} \\
& =R_{i}(\theta)\left\{1+\xi\left(\frac{-2 \gamma+3}{R_{i}^{3}(\theta) \eta_{\theta}^{2}(\theta)}\right)\right\}^{\frac{1}{-2 \gamma+3}}
\end{aligned}
$$

and for $\gamma=1.5$ gives

$$
R_{o}(\theta)=R_{i}(\theta) \exp \left(\frac{\xi}{R_{i}^{3}(\theta) \eta_{\theta}^{2}(\theta)}\right)
$$

where $\xi$ is referred to as the 'density-luminosity parameter' and is a constant of the nebula model quantifying both the stellar luminosity and inner equatorial density.

The intensity of emitted light at a point within the nebula is proportional to the square of the density of radiators at that point. The nebula is assumed to be optically thin at visible wavelengths; consequently, all the emitted light escapes from the nebula. The image $I(x, y)$ is computed numerically by integrating the squared density along the line-of-sight. Figure 3 shows each model's estimate of the PN known as IC418 and a difference image to compare the IBPES model to the data. 


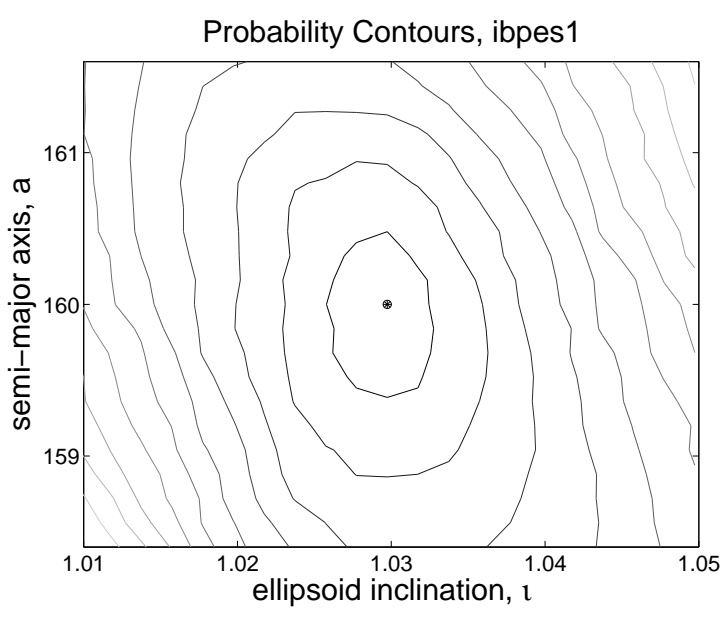

FIGURE 4. Contours of $\boldsymbol{\imath}$ vs. $a$ for Synthetic PN ibpes1. The apparent semi-major axis $\hat{a}$ is approximately $b+(a-b) \sin \imath$. The true solution is marked by a ' $\bullet$ '. Inclination is in radians.

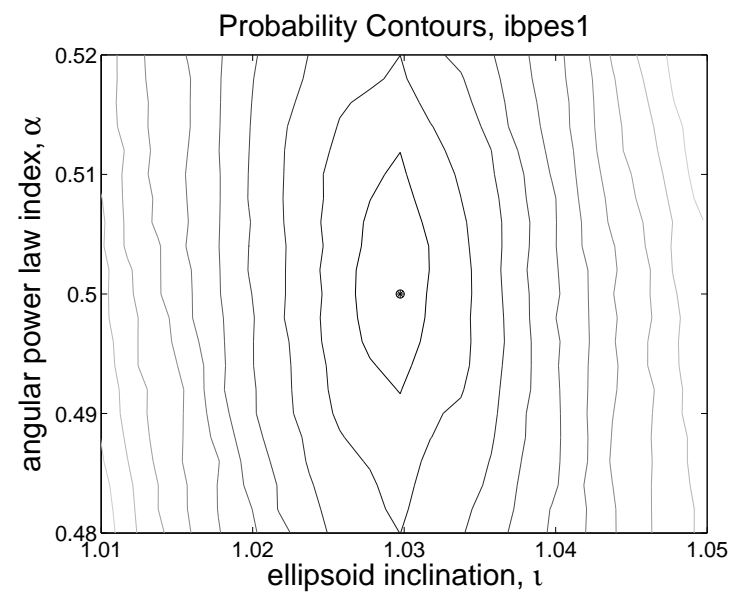

FIGURE 5. Contours of $\imath$ vs. $\alpha$ for Synthetic PN ibpes 1 . The ' $\bullet$ ' marks the true solution. Inclination is in radians. Both $\alpha$ and $\imath$ can affect limb brightening but are distinguishable nevertheless.

\section{Bayesian Estimation}

Let $M$ represent the model-generated image ('model') and $D$ represent the actual HST image ('data'). We apply Bayes' theorem

$$
\operatorname{prob}(M \mid D, I) \propto \operatorname{prob}(D \mid M, I) \cdot \operatorname{prob}(M, I),
$$

and assign uniform priors. The likelihood is based upon the difference image $(M-D)$, which is an $X \times Y$ array of pixel-wise differences $\left(M_{x y}-D_{x y}\right)$. Assuming the pixels are i.i.d., we get

$$
\operatorname{prob}(D \mid M, I) \propto \exp \left[-\frac{(M-D)^{2}}{\sigma^{2}}\right]=\exp \left[-\sum_{x=1}^{X} \sum_{y=1}^{Y} \frac{\left(M_{x y}-D_{x y}\right)^{2}}{\sigma^{2}}\right] .
$$

Taking the log of both sides yields

$$
\log \operatorname{prob}(D \mid M, I) \propto\left[-\sum_{x=1}^{X} \sum_{y=1}^{Y} \frac{\left(M_{x y}-D_{x y}\right)^{2}}{\sigma^{2}}\right] .
$$

Analytic expressions for the log probability do not exist for the IBPES model and must be computed numerically. A typical cropped HST image is $350 \times 400$ pixels. The 3D model is $350 \times 400 \times 400$ voxels. On a $1.2 \mathrm{GHz}$ processor, one image can be constructed in five minutes. Thus far, $\omega, y_{o}$, and $z_{o}$ have exhibited only trivial changes during 11parameter IBPES searches. ${ }^{1}$ Eight parameters $\left(I_{o}, a, b, \imath, \alpha, \beta, \gamma, \xi\right)$ would remain if three were eliminated, requiring 45 minutes for each calculation of the gradient vector. If not eliminated, eleven parameters remain and 60 minutes are required for each vector.

${ }^{1}$ Parameters 12 and 13 - expansion velocity and distance to earth — are not yet incorporated into a model. 


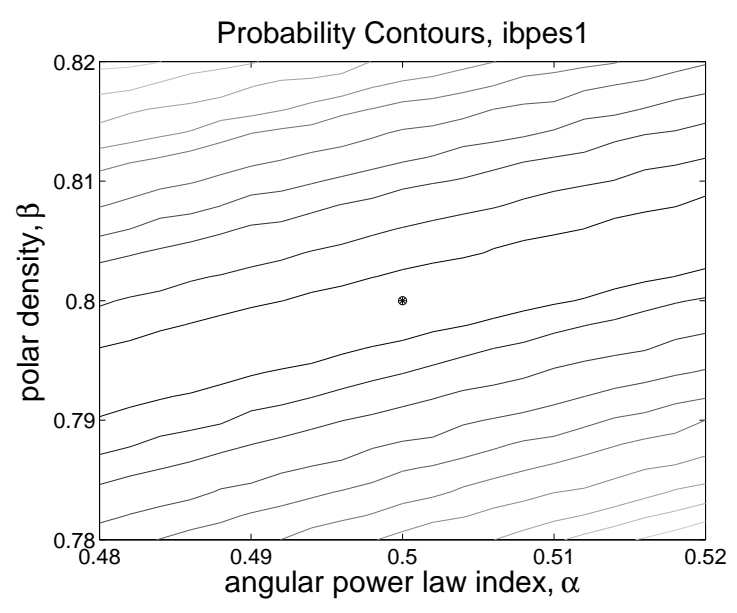

FIGURE 6. Contours of $\alpha$ vs. $\beta$. These two parameters are strongly correlated. Both of them influence the area and amplitude of the bright equatorial limbs in the IBPES image: Either a larger $\alpha$ or a smaller $\beta$ makes the bright limb more compact.

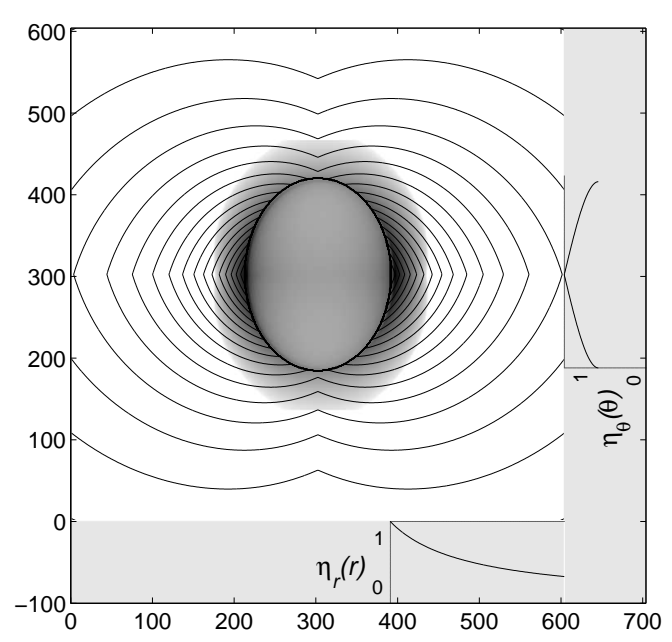

FIGURE 7. Density contours for IC418, unrotated and viewed side-on. In grey along the $\mathrm{X}$-axis, the radial density function $\eta_{r}(r)$ peaks at the equator and falls as $r^{-\gamma}$. Along the y-axis, the latitudinal density function $\eta_{\theta}(\theta)$ peaks at the equator and falls as $(2 \theta / \pi)^{\alpha}$.

\section{PRELIMINARY RESULTS}

The IBPES model is defined such that, at inclination $\imath=0$, one looks down the long axis of the prolate spheroid and sees a circular nebula profile. This profile elongates with inclination until maximum elongation at an inclination of $\pi / 2$. The apparent semimajor axis $\hat{a}$ is approximately related to $l$ and to the true semi-major axis $a$ through the relation $\hat{a}=b+(a-b) \sin \imath$, where $b$ is the semi-minor axis. It is not obvious that $a$ and $\imath$ can be learned directly. We demonstrate that they can be learned by using a synthetic nebula, ibpes1. (See Figure 2.) Note that the contour plot of $l$ vs. $a$ in Figure 4 exhibits a well-defined minimum. Ibpes1 is quite elongated, having an eccentricity above 0.7 . Additional study is required in the case of nearly circular nebulae.

A contour plot was constructed for each pair of model parameters using ibpes1. All plots but one exhibit a distinct minimum. Figure 5 provides an example of the minimum for $l$ versus $\alpha$. Both of these parameters influence the size and shape of the bright regions on the rim of the nebula image. These quantities are also distinguishable in the case of elongated nebulae.

The exceptional contour plot, shown as Figure 6 , relates $\alpha$ and $\beta$ and reveals that they are correlated. Recall from (10) that $\alpha$ and $\beta$ are parameters of the latitudinal density function $\eta_{\theta}(\theta)$. As it happens, both $\alpha$ and $\beta$ control the 'peakiness' of the rim brightening: An increase in $\alpha$ causes the bright region to become more compact, and a decrease in $\beta$ does the same. We are exploring a re-formulation of the latitudinal density function that avoids these difficulties.

One benefit of learning a model of the nebula is that we have the ability to alter the rotation of the model and, thereby, view a PN from any angle and at any distance. Figure 7 shows a preliminary model of IC418 surrounded by density contours. Also 
shown (bottom) is a plot of $\eta_{r}(r)$, the radial density function, and (right) a plot of $\eta_{\theta}(\theta)$, the latitudinal density function. The densities peak at the equator on the surface of the inner ellipsoidal bubble. This is due to a higher density of gas along the nebula's equator.

\section{CONCLUSIONS}

The hierarchy has proven to be a powerful tool to enable a more rapid estimation of the parameters in a very difficult forward problem. However, IBPES has proven to be quite sensitive to initial conditions, unlike earlier stages. IBPES initial conditions are generated by translating the FastSES solution into IBPES variables, but the FastSES solution does not capture enough information to generate robust initial conditions for IBPES. Altering the hierarchy to determine more parameters might improve the situation.

Gradient descent is currently the only search method implemented for the entire hierarchy. IBPES search is also implemented using simplex search in AutoBayes [6]. When initialized to the FastSES solution, simplex produces results similar to gradient descent. Cursory explorations with IC418 reveal problems with both gradient descent and simplex methods, which may be solved with more sophisticated search methods.

\section{ACKNOWLEDGMENTS}

The NASA Ames team was supported by NASA Ames DDF Grant 274-51-02-51, the NASA IDU/IS/CICT Program and the NASA Aerospace Technology Enterprise. The USNO team was supported by NASA grants GO7501, GO8390, GO8773 through the Space Telescope Science Institute, operated by AURA, Inc. under NASA contract NAS5-26555. Thanks also to Steven Movit (Penn State) for significant past efforts.

\section{REFERENCES}

1. Hajian, A. R., Movit, S. M., Balick, B., Terzian, Y., Palen, S., Knuth, K. H., Bond, H., and Panagia, N., ApJ Supp. (2004), submitted.

2. Knuth, H., K., and Hajian, A. R., "Hierarchies of models: toward understanding planetary nebulae.," in Bayesian Inference and Maximum Entropy Methods in Science and Engineering, edited by C. J. Williams, American Institute of Physics, Melville, NY, 2002, vol. 659, pp. 92-103, 22nd International Workshop, Moscow, ID.

3. Aaquist, O. B., and Kwok, S., ApJ, 462, 813 (1996).

4. Masson, C. R., ApJ, 346, 243-250 (1989).

5. Masson, C. R., ApJ, 348, 580-587 (1990).

6. Fischer, B., Hajian, A. R., Knuth, K. H., and Schumann, J., "Automatic derivation of statistical data analysis algorithms: Planetary nebulae and beyond."' in Bayesian Inference and Maximum Entropy Methods in Science and Engineering, edited by Y. Zhai and G. J. Erickson, American Institute of Physics, Melville, NY, 2003, vol. 707, pp. 276-294, 23rd International Workshop, Jackson Hole, WY. 